\title{
Desempenho de organizações policiais: revisão sistemática da literatura
}

\section{Andersson Pereira dos Santos}

Polícia Federal (PF), Brasília - DF, Brasil

\section{Adalmir de Oliveira Gomes}

Universidade de Brasília (UnB), Brasília - DF, Brasil

Em razão da relevante função que as polícias cumprem na sociedade, é importante a obtenção de informações que possam ser utilizadas para avaliar o seu desempenho e, com isso, aprimorar seus serviços e atender às exigências e necessidades da comunidade. Nesse sentido, o presente trabalho teve como objetivo efetuar uma revisão sistemática da literatura acerca do desempenho de organizações policiais. Foram revisados artigos teórico-empíricos publicados entre 2010 e 2020 que tinham como propósito avaliar ou mensurar o desempenho de polícias. Buscou-se identificar modelos teórico-empíricos, com instrumentos de coleta de dados, métodos de análise de dados e variáveis que são empregados em pesquisas a respeito do desempenho das polícias. Por meio dos resultados da revisão de literatura, verificou-se que, dada a complexidade e a diversidade dos serviços prestados pelas organizações policiais, não é possível o estabelecimento de metodologia única para que seja realizada a avaliação do seu desempenho. Por essa razão, o desempenho dessas organizações deve ser mensurado de acordo com os objetivos e atribuições de cada polícia que será avaliada, conforme as atividades que são realizadas e em consonância com a dimensão que se deseja medir. Somente após essa definição é possível escolher as variáveis que serão utilizadas para analisá-lo. Com base nos resultados encontrados, é proposta uma agenda de pesquisa com a finalidade de definir e avaliar o desempenho das polícias brasileiras, de acordo com as suas características e atribuições, de modo a possibilitar o aumento do controle social sobre essas organizações e a melhoria do seu desempenho.

Palavras-chave: polícia, desempenho policial, revisão sistemática da literatura 


\section{Desempeño de las organizaciones policiales: revisión sistemática de la literatura}

Debido al papel relevante que tiene la el trabajo de la policía en la sociedad es importante obtener información que pueda usarse para evaluar su desempeño y, con esto, mejorar sus servicios y cumplir con los requisitos y necesidades de la comunidad. En este sentido, el presente estudio tiene como objetivo realizar una revisión sistemática de la literatura sobre el desempeño de las organizaciones policiales. Artículos teórico-empíricos con el fin de evaluar la medición del desempeño policial, publicados entre 2010 y 2020, fueron revisados. Se buscó identificar modelos teórico-empíricos, con instrumentos de recolección de datos, métodos de análisis de datos y variables, que se utilizan en investigación sobre desempeño policial. A través de los resultados de la revisión de la literatura, se encontró que, dada la complejidad y diversidad de los servicios prestados por las organizaciones policiales, no es posible establecer una metodología única para que se evalúe su desempeño. Por esta razón, el desempeño de estas organizaciones debe medirse en función de los objetivos y funciones de cada policía a evaluar, de acuerdo con las actividades que se realizan y en línea con la dimensión que se desea medir. Sólo después de esta definición es posible elegir las variables que serán utilizadas para analizarlo. Con base en los resultados encontrados, se propone una agenda de investigación con el propósito de definir y evaluar el desempeño de la policía brasileña, de acuerdo con sus características y atribuciones, con el fin de permitir incrementar el control social sobre estas organizaciones y la mejora de su desempeño.

Palabras clave: policía, desempeño de la policía, revisión sistemática de la literatura

\section{Performance of police organizations: systematic literature review}

Given the significant role that the police fulfill in society it is important to obtain information that can be used to evaluate their performance and, thus, improve their services and meet the demands and needs of the community. In this sense, the present study aimed to carry out a systematic review of the literature about the performance of police organizations. Theoretical and empirical articles published between 2010 and 2020 that aimed to evaluate or measure the performance of police organizations were reviewed. Searched to identify theoretical-empirical models, with data collection instruments, data analysis methods and variables, which are used in research regarding police performance. Throughout the results of the literature review, it was found that, given the complexity and diversity of services provided by police organizations, it is not possible to establish a single methodology for the performance assessment to be carried out. For this reason, the performance of these organizations should be measured in accordance with the objectives and duties of each police that will be evaluated, according to the activities that are carried out and in line with the dimension that is to be measured. Only after this definition is it possible to choose the variables that will be used for the analysis. Based on these results, a research agenda is proposed to define and evaluate the performance of Brazilian police, according to their characteristics and duties, to enable the increase of social control over these organizations and improve their performance.

Keywords: police, police performance, systematic literature review 


\section{Introdução}

A sociedade aspira conhecer medidas de desempenho confiáveis das organizações policiais para garantir que a polícia esteja gerando resultados com os recursos recebidos (PINC, 2009). Conhecer o desempenho das polícias é necessário para satisfazer as exigências de prestação de contas externas e para o estabelecimento de responsabilização dentro das organizações (DURANTE; BORGES, 2011).

Nesse sentido, para que sejam atingidos os propósitos da segurança pública, se faz necessário o aperfeiçoamento dos controles interno e externo das organizações que atuam nesta área (SOUZA; REIS, 2007). Dotar de informações pesquisadores e gestores sobre o desempenho das organizações policiais, com indicadores claros e objetivos, respaldados no método científico, ajudaria o Estado a aprimorar a gestão da segurança pública, tendo em vista que não é possível gerenciar aquilo que não é medido (VIEIRA; PROTÁsIO, 2011; FERREIRA; RIGUEIRA, 2013).

Sob essa perspectiva, o presente trabalho busca contribuir em um campo do conhecimento que carece de estudos empíricos no Brasil. O artigo apresenta uma revisão sistemática da literatura, com o levantamento dos estudos teórico-empíricos publicados entre 2010 e 2020 acerca do desempenho de organizações policiais. Os resultados podem auxiliar pesquisadores, profissionais e gestores no processo de avaliação do desempenho das polícias brasileiras, buscando com isso a melhoria dos serviços prestados por tais organizações.

\section{Fundamentos teóricos}

\subsection{Desempenho de organizações policiais}

Com o incremento das atividades policiais, ocorrido em decorrência do aumento da criminalidade, bem como em razão da relevante função que as organizações policiais possuem para a segurança e, consequentemente, para a qualidade de vida dos cidadãos, é de suma importância a obtenção de informações que possam ser utilizadas para avaliar o 
seu desempenho (CARMONA; GRÖNLUND, 2003). A polícia, assim como outras organizações públicas, está procurando um modelo acessível, responsável, sustentável e ético para sua atuação (LELANDAIS, 2007). O elemento mais tangível desse cenário é o crescente uso de medidas, particularmente a adoção de indicadores-chave de desempenho, que se tornou cada vez mais frequente no âmbito da polícia e das demais organizações da segurança pública (ROCHE, 2008).

Analisar o desempenho de organizações policiais significa examinar conjuntos de indicadores, procedimentos internos, instrumentos e processos de prestação de contas internos e externos, através dos quais o desempenho é definido, avaliado e monitorado (MAIllaRd; SAVAge, 2018). A avaliação do desempenho policial permite conciliar os objetivos do seu serviço com os meios implementados e os resultados alcançados (LELANDAIS, 2007).

Em razão da discricionariedade inerente à função policial, monitorar o trabalho cotidiano dos policiais é um desafio para todas as organizações de segurança pública do mundo (CANO; DUARTE, 2014). O serviço prestado pelas polícias representa uma área complexa do serviço público, em razão da diversidade das atividades que são desempenhadas e da ausência de consenso sobre quais devem ser os seus objetivos prioritários (COLLIER, 1998). Não existe concordância quanto à forma ou à natureza do que deve ser medido, qual medida deva ser utilizada, o que diferentes indicadores significam e como eles serão utilizados para promover a melhoria do desempenho (TIWANA; BASS; FARREL, 2015). Como reflexo dessa ausência de consenso, a avaliação de desempenho policial se torna ambígua e complexa (VolLARD, 2003).

A natureza do trabalho das polícias implica que, enquanto as suas medidas de desempenho podem coincidir com as de outras organizações, elas também variam significativamente em razão das diferentes questões metodológicas e substantivas no seu modo de atuar (TIWANA; BASS; FARREL, 2015). A complexidade, a heterogeneidade, a diversidade e a incerteza são características dos serviços policiais que afetam diretamente o seu desempenho (CARTER; KLEIN; DAY, 1993) e tornam difícil o estabelecimento de uma metodologia para que seja realizada a avaliação do seu desempenho (ColLIER, 2001). Tal constatação ressalta a importância e a necessidade de se desenvolver métodos próprios para que seja implementado um efetivo sistema de avaliação do desempenho policial 
(HOLMES; PIÑERES; KIEL, 2006), devendo ser construído de acordo com as peculiaridades de cada organização, com um conjunto de indicadores específicos que serão aplicados às atividades por ela realizadas (DOOREN, 2005).

Por essa razão, a avaliação de desempenho de uma organização policial deve ser iniciada com a precisa demarcação dos objetivos e das atividades-chave que serão avaliadas (ROGERSON, 1995). Para a realização dessa avaliação, deve ser estabelecido o conjunto de indicadores de desempenho que será aplicado, como, por exemplo, taxa de crimes, número de chamadas por unidade policial, satisfação da população em relação ao desempenho e ao serviço prestado pela organização policial, entre outros (HoQUE; ARENDS; AlexANDER, 2004). O estabelecimento de medidas de desempenho e a aplicação de métodos de avaliação constituem um estímulo à melhoria do desempenho nos diferentes âmbitos de atuação das polícias (VOLLARD, 2003), o que facilita o início de uma cultura de avaliação, que é incipiente na administração pública e, em especial, nas forças policiais (COLLIER, 2001).

\subsection{Dimensões do desempenho de organizações policiais}

Uma questão central para a avaliação do desempenho de organizações policiais é o estabelecimento das dimensões em torno das quais ele pode ser medido, avaliado e comparado (MAILlARD; SAVAGE, 2012). As dimensões do desempenho da polícia são complexas e assumem diferentes formas, em parte porque não há consenso sobre o que constitui desempenho ou como esse deve ser medido (MAGUIRE, 2004). Isso pode resultar de diferenças nas expectativas e prioridades sociais e políticas sobre o que a polícia deve alcançar, bem como em razão de vários mitos associados ao papel e função da polícia (SHANE, 2013).

De acordo com Shane (2013), a função de aplicação da lei, que é fazer com que as leis sejam cumpridas, geralmente é a principal preocupação dos gestores de organizações policiais, de modo que as dimensões do desempenho como, por exemplo, a qualidade ou a eficiência, ficam ignoradas. Por essa razão, a adoção de medidas que reflitam as múltiplas dimensões do desempenho policial promove uma abordagem equilibrada (SHANE, 2013). Segundo o autor, esse método é susceptível de satisfazer várias partes interessadas, como a própria polícia, os cidadãos e os governantes, o que é fundamental para criar 
um ambiente harmonioso, podendo reduzir o estresse e introduzir mais racionalidade na organização policial, contribuindo para a melhoria do seu desempenho.

Normalmente, os departamentos de polícia usam vetores de insumos, como, por exemplo, mão de obra, veículos, aparelhos, armas e edifícios para produzir um vetor de resultados, como a prevenção e detecção de crime, manutenção de lei e ordem, regulamentação e controle de trânsito, segurança de dignitários, entre outros (DRAKE; SIMPER, 2005). Segundo os autores, os produtos estão sob o controle de departamentos policiais, enquanto os resultados são objetivos socialmente desejáveis, que podem ser influenciados pelos fatores que não estão sob controle da polícia. Dado esse conjunto complexo de vetores de entrada e saída/resultado, definir a eficiência da polícia é um grande desafio e, geralmente, existe sobreposição entre os produtos e os resultados dos serviços policiais (DAVIS, 2012).

Os pesquisadores, frustrados com as medidas convencionais de desempenho da polícia (taxas de criminalidade, prisões, autorizações, entre outros), têm incluído medidas de qualidade de vida e equidade em desempenho policial (MIRZER, 1996). Como resultado, tanto os pesquisadores como os profissionais que integram as organizações policiais têm incentivado uma maior atenção aos processos de policiamento que refletem valores democráticos compartilhados, em vez de simplesmente contar atividades ou resultados (TYLER, 2001), buscando novas medidas da qualidade do serviço policial (ROSENBAUM; LAWRENCE; HARTNETT; MCDEVITT; POSICK, 2015).

A natureza multidimensional do trabalho policial exige, naturalmente, uma abordagem multidimensional da medição do seu desempenho (MASLov, 2014). Nesse sentido, alguns pesquisadores sugerem que o desempenho da polícia deve ser medido por múltiplos indicadores, que vão desde a contagem individual das vítimas (NEYROUD, 2008) até pesquisas de satisfação pública mais formalizadas. Um dos estudos mais citados na literatura sobre o quadro multidimensional do trabalho policial foi feito por Moore e Braga (2003), que identificaram sete dimensões do trabalho policial, apresentadas no Quadro 1. 
Quadro 1 | Dimensões do desempenho policial

\begin{tabular}{|l|l|}
\hline Nome & Conceito \\
\hline $\begin{array}{l}\text { Reduzir o crime e vitimização } \\
\text { (Reduce crime and criminal victimization). }\end{array}$ & Reduzir a taxa de criminalidade. \\
\hline $\begin{array}{l}\text { Chamar os infratores para prestar contas } \\
\text { (Call offenders to account). }\end{array}$ & $\begin{array}{l}\text { Taxa de apuração de crimes e } \\
\text { responsabilização dos infratores } \\
\text { (crimes resolvidos, infratores presos). }\end{array}$ \\
\hline $\begin{array}{l}\text { Reduzir o medo e aumentar a segurança pessoal } \\
\text { (Reduce fear and enhance personal security). }\end{array}$ & $\begin{array}{l}\text { Redução do medo e aumento da } \\
\text { sensação de segurança. }\end{array}$ \\
\hline $\begin{array}{l}\text { Garantir a segurança e civilidade nos espaços } \\
\text { públicos (Ensure civility in public spaces). }\end{array}$ & $\begin{array}{l}\text { Assegurar a segurança no trânsito e em } \\
\text { espaços públicos. }\end{array}$ \\
\hline $\begin{array}{l}\text { Uso da força e autoridade de forma justa, eficiente e } \\
\text { efetiva (Use force and authority fairly, efficiently, and } \\
\text { effectively). }\end{array}$ & $\begin{array}{l}\text { Usar a autoridade e força de maneira } \\
\text { justa e proporcional. }\end{array}$ \\
\hline $\begin{array}{l}\text { Uso de recursos de forma eficiente e razoável. } \\
\text { (Using resources efficiently and fairly). }\end{array}$ & $\begin{array}{l}\text { Utilizar recursos de forma razoável } \\
\text { (eficiência, produtividade, efetividade). }\end{array}$ \\
\hline $\begin{array}{l}\text { Qualidade do serviço/satisfação do cidadão (Quality } \\
\text { services/customer satisfaction). }\end{array}$ & $\begin{array}{l}\text { Qualidade do serviço e a satisfação do } \\
\text { cidadão. }\end{array}$ \\
\hline
\end{tabular}

Fonte: adaptado de Moore e Braga (2003).

Não há uma única medida de desempenho que possa avaliar todas as dimensões do trabalho policial ao mesmo tempo, tampouco existe uma medida perfeita que possa avaliar uma única dimensão do trabalho policial (GALLAGHER; MAGUIRE; MASTROFSKI; REISIG, 2001). As sete dimensões identificadas por Moore e Braga (2003) podem ser usadas com a finalidade de se estabelecer uma estrutura para medir o desempenho da polícia (Kiedrowski; Petrunik; MAcdonald; Melchers, 2013).

\section{Procedimentos metodológicos}

O objetivo da revisão sistemática da literatura é identificar o atual estágio do conhecimento a respeito de uma determinada área da ciência (LACERDA; ENSSLIN; ENSSLIN, 2012). Para os acadêmicos, o processo de revisão sistemática aumenta o rigor metodológico do levantamento da literatura e, para profissionais e gestores, a revisão 
sistemática ajuda a desenvolver uma base de conhecimento confiável, acumulada de uma série de estudos realizados (TRANFIELD; DENYER; SMART, 2003), que pode ser utilizada para o aprimoramento das atividades nas organizações. Nesse sentido, a revisão sistemática da literatura realizada teve o objetivo de identificar o que de mais recente e relevante foi pesquisado a respeito do desempenho policial, por meio do levantamento dos estudos teórico-empíricos publicados entre 2010 e 2020 acerca do tema. Foram aplicadas as setes etapas propostas por Fink (2014), visualizadas na Figura 1.

Figura 1 | Etapas para a realização de revisão sistemática da literatura, propostas por Fink (2014)

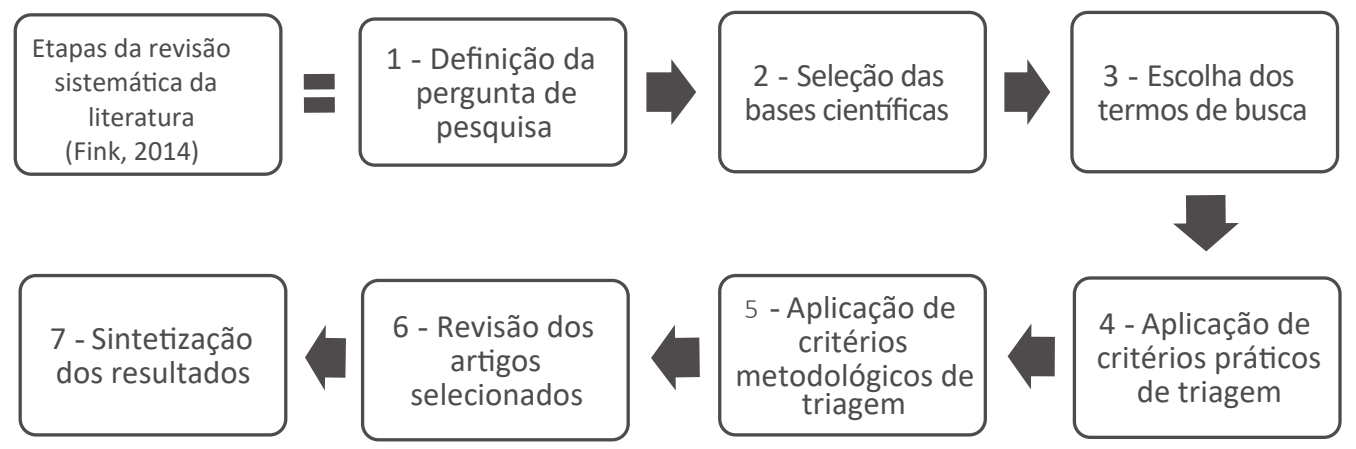

Fonte: adaptado de Fink (2014).

Na primeira etapa foi definida a pergunta de pesquisa: "como o desempenho de organizações policiais está sendo avaliado? " Para responder a essa pergunta foram selecionadas as bases científicas Scopus e Web of Science, que indexam e sumarizam mais de 15 mil periódicos científicos internacionais, contendo a maioria das citações importantes para a elaboração de artigos (BAKKALBASI; BAUER; GLOVER; WANG, 2006). No levantamento de artigos produzidos em língua portuguesa, foram selecionadas, além das bases informadas, a Spell e Scielo, que concentram e divulgam pesquisas nacionais, especialmente nas áreas de administração e ciências sociais. Dessa maneira, entendese que as bases selecionadas se constituem naquelas de maior relevância para o tema pesquisado.

Em seguida, foram escolhidos os termos de busca (palavras-chave). No levantamento preliminar, utilizando a palavra "performance" associada a "police" ou a variante "policing", os resultados encontrados nas bases Scopus e Web of Science apresentaram centenas de 
artigos em inglês, tratando de diversas temáticas e não apenas do desempenho policial. Com o intuito de selecionar estudos com uma amostragem mais representativa, foram utilizadas as palavras-chave mais citadas em artigos que efetivamente realizaram avaliação de desempenho de organizações policiais, indicadas nos sistemas de busca das bases consultadas. Para o levantamento em língua portuguesa foi utilizada a palavra-chave "desempenho" associada à "polícia" e "policial". Em razão do baixo número de artigos encontrados, foram adicionados vocábulos traduzidos entre os mais citados nas pesquisas publicadas em inglês a respeito do tema, como "indicadores", "eficiência" e "eficácia". As palavras-chave utilizadas na revisão estão indicadas no Quadro 2.

\section{Quadro 2 | Palavras-chave utilizadas na revisão de literatura}

\begin{tabular}{|c|c|}
\hline Inglês & Português \\
\hline "police (policing) performance” & polícia (policial) E desempenho \\
\hline police (policing) AND “performance measure” & polícia (policial) E indicadores \\
\hline police (policing) AND “performance management” & polícia (policial) E indicador \\
\hline police (policing) AND “performance measurement” & polícia (policial) E eficiência \\
\hline police (policing) AND “performance indicators” & polícia (policial) E produtividade \\
\hline police (policing) AND “performance metrics” & polícia (policial) E eficácia \\
\hline police (policing) AND “performance evaluation” & \\
\hline police (policing) AND “performance assessment” & \\
\hline
\end{tabular}

Fonte: elaborado pelos autores.

Na quarta etapa foram estabelecidos os critérios práticos para triagem. Foi definido que seriam utilizados apenas artigos revisados por pares, publicados em periódicos científicos entre 2010 e 2020 (abril), para que se obtivesse o que de mais recente tenha sido pesquisado sobre o tema desempenho policial, em língua inglesa ou portuguesa. Também foi delimitado que a busca seria feita no título, palavras-chave e resumo dos artigos (TÓPICO / TITLE-ABS-KEY). Com base nesses critérios, foram identificados 339 artigos na base Scopus, 243 na Web of Science, 33 na Scielo e nove na Spell. Após a exclusão dos artigos duplicados, que se repetiram nas bases, remanesceram 369 artigos em inglês e 35 artigos em língua portuguesa para serem analisados. 
A quinta etapa foi dividida em três momentos. Inicialmente, foi feita a leitura do resumo de todos os 404 artigos. Foram descartados 234 artigos cujos resumos não tratavam de desempenho policial, mas, sim, de outros temas sem qualquer relação com a polícia ou nos quais a polícia foi utilizada como locus para analisar outros fenômenos que não o seu desempenho. O Quadro 3 apresenta exemplos de estudos que foram descartados no primeiro momento da quinta etapa:

\section{Quadro 3 | Exemplos de artigos descartados no primeiro momento da quinta etapa da revisão de literatura}

\section{Objetivo do artigo}

Motivo do descarte

Comparar o desempenho de segurança de grades de proteção

W-post G4 (1S) e barreiras de cabos instaladas nas medianas Objeto não trata da polícia.

de rodovias na Flórida.

Examinar a adesão dos médicos do trabalho (OP) à diretriz holandesa sobre o gerenciamento de problemas comuns de saúde mental.

Avaliar o nível de drogas em motoristas abordados pela polícia.

Objeto não trata da polícia.

Avaliar o stress de cavalos policiais.

Polícia utilizada como locus para estudar outro fenômeno.

Polícia utilizada como locus para estudar outro fenômeno.

Avaliar a aptidão física de policiais

Polícia utilizada como locus para estudar outro fenômeno.

Fonte: elaborado pelos autores.

$\mathrm{Na}$ sequência, foram escolhidos e aplicados critérios metodológicos de triagem. Foi feita a leitura integral de 170 artigos, 165 em língua inglesa e 5 em português. Foram selecionados artigos teórico-empíricos para serem revisados. Foram considerados artigos teórico-empíricos os trabalhos que apresentaram resultados de estudos ou pesquisas, fundamentados em diferentes perspectivas teóricas e metodológicas (ENANPAD, 2020). A opção por utilizar trabalhos teórico-empíricos na revisão foi decorrente da busca por identificar modelos teórico-empíricos de análise do desempenho policial, com instrumentos de coleta de dados, métodos de análise de dados e variáveis próprios, que possam ser empregados em pesquisas futuras a respeito do desempenho das polícias brasileiras. 
Foram descartados os artigos que não analisavam o fenômeno desempenho, embora citassem o termo no título, resumo ou palavras-chave relacionado ao exercício de alguma atividade policial, bem como os ensaios teóricos, que consistem na discussão do tema a partir do posicionamento teórico do (s) autor (es) em relação a uma ou várias teorias abordadas e não apresentam resultados de pesquisas (ENANPAD, 2020). O Quadro 4 apresenta exemplos dos estudos que foram descartados do segundo momento da quinta etapa.

\section{Quadro 4 | Exemplos de artigos descartados no segundo momento da quinta etapa da revisão de literatura}

\section{Objetivo de artigo}

Apresentar a arquitetura de um Sistema Inteligente de Apoio à Decisão para cumprir as responsabilidades emergentes das agências policiais.

Analisar variáveis gerenciais, profissionais e demográficas que influenciam o comprometimento organizacional dos policiais.

Nesta peça conceitual, argumentamos que a abordagem atual para medir o desempenho da polícia normalmente baseada no uso de métricas policiais tradicionais não alcançou os resultados desejados e que é necessária uma estratégia diferente.

Este artigo considera como o desempenho da polícia pode ser aprimorado com a adoção da filosofia "Lean", um potencial propulsor de melhorias policiais internas.

\section{Motivo do descarte}

Não analisou desempenho.

Não analisou desempenho.

Ensaio teórico Fonte: elaborado pelos autores

Assim, restaram 69 artigos teórico-empíricos que tinham entre seus objetivos avaliar o desempenho de alguma organização policial, em qualquer país. Desses, apenas um artigo em língua portuguesa. Ainda na execução da quinta etapa, para garantir a relevância dos artigos encontrados, foi feita a avaliação de impacto dos periódicos onde foram publicados. Para avaliação dos periódicos estrangeiros, foi escolhido o Scimago Journal Rank (SJR), indicador bibliométrico que mede o prestígio ou a influência de uma revista científica, calculada por meio de uma janela de citação de três anos, amplo o suficiente para incluir a maioria das citações, e dinâmico o bastante para medir a evolução das revistas científicas (González-Pereira; Guerrero-Bote; Moya-Anegón, 2010). A consulta ao SJR foi realizada em abril de 2020. 
O SJR divide os periódicos em quatro quartis (Q1, Q2, Q3 e Q4), ranqueados de acordo com o seu impacto, do mais relevante (Q1) até aqueles com menor influência (Q4). Optou-se por utilizar apenas os 50 artigos publicados em periódicos avaliados como "Q1" e os dez trabalhos divulgados em revistas qualificadas como "Q2", que concentram os $50 \%$ periódicos mais bem classificados. Foram descartados sete artigos publicados em periódicos avaliados como "Q3" e o trabalho divulgado em revista posicionada em "Q4".

O artigo em língua portuguesa foi mantido para análise em razão da classificação obtida no Qualis-Periódicos, revista na qual foi publicado. O periódico, qualificado como "B1" em administração, encontra-se entre os estratos que concentram os $50 \%$ melhores classificados no Brasil (CAPES, n. d.), mantendo a simetria com o critério aplicado aos artigos publicados em língua inglesa. Assim, com base nesse recorte metodológico, fundamentado nas etapas propostas por Fink (2014) para realizar o processo de revisão sistemática da literatura, foram selecionados 61 artigos.

Por último, foi executada a sexta etapa, a revisão propriamente dita. Os artigos foram separados por ano de publicação. Em seguida, foram elaborados os aspectos a serem analisados, a fim de entender como os estudos a respeito do desempenho de organizações policiais estão sendo tratados. Para tanto, buscou-se identificar os países onde foram aplicadas as pesquisas, periódicos em que foram publicadas, natureza dos estudos, instrumentos de coleta de dados utilizados, origem dos dados, níveis de análise, técnicas de análises de dados aplicadas e dimensões do desempenho policial estudadas, de acordo com a classificação proposta por Moore e Braga (2003). A sintetização dos resultados, sétima e última etapa (FINK, 2014), com as variáveis mais utilizadas e os principais resultados de pesquisa, será apresentada na próxima seção.

\section{Resultados}

\subsection{Visão geral dos artigos revisados}

A produção de artigos teórico-empíricos a respeito do desempenho policial foi, em média, de seis artigos por ano, tendo sido constatada uma flutuação do número de 
publicações ao longo da última década (2010/2019), como pode ser visualizada na Figura 2. Em alguns anos muitas pesquisas foram divulgadas, como em 2014 e 2018 (11 artigos) e 2019 (8 artigos), e em outros anos poucos trabalhos teórico-empíricos a respeito do tema foram publicados, como em 2016 (2 artigos) e 2011 (sem registro). A média de artigos publicados reflete a tendência crescente do "gerencialismo" e uso de métodos científicos para medir o desempenho na polícia (ASIF et al., 2018), apesar da dificuldade da medição (HADAD; KEREN; HANANI, 2015) e da insuficiência de dados para avaliar o desempenho de unidades policiais (TOMAŽEviČ; SELJAK; ARISTOVNIK, 2016). No ano de 2020, até abril, apenas um artigo havia sido publicado sobre o tema (DESMOND; REYNS; FRANK; KLAHM IV; HENSON, 2020).

Figura 2 | Publicações a respeito do desempenho policial na década de 2010/2019

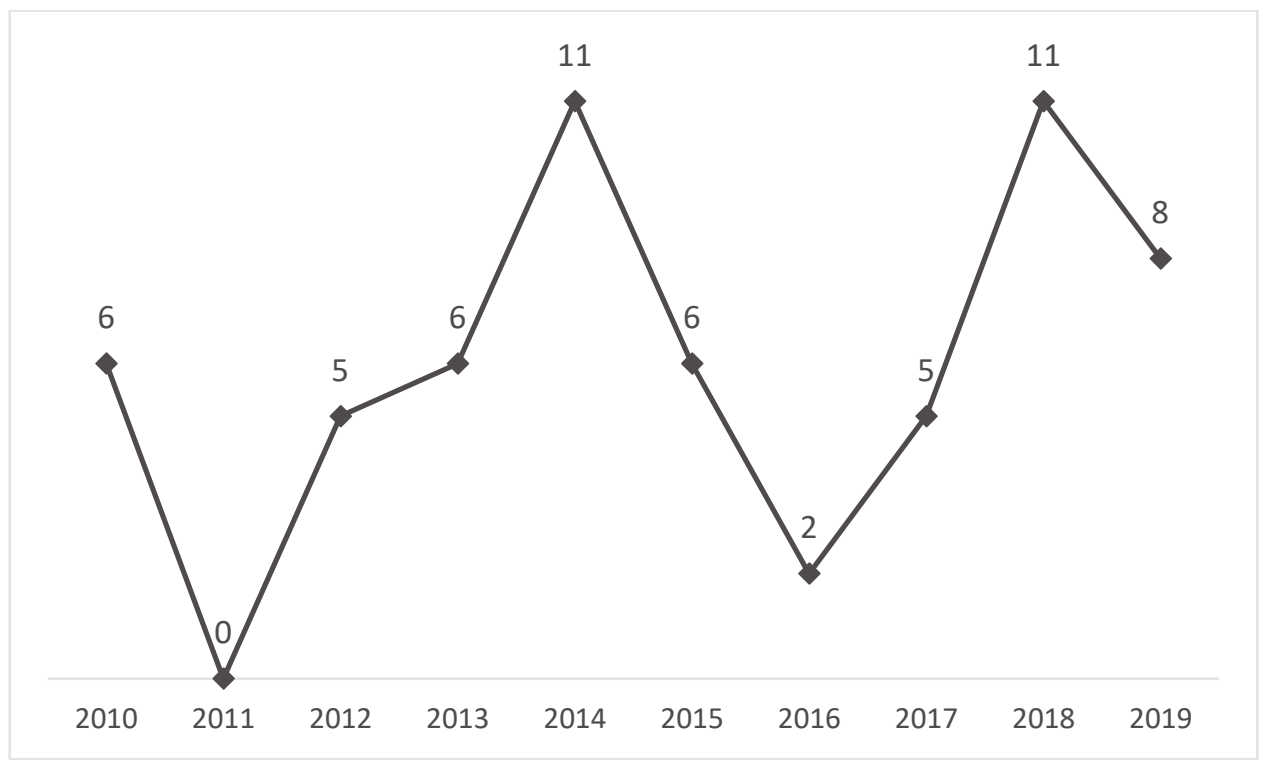

Fonte: elaborado pelos autores.

No tocante aos países onde as pesquisas foram realizadas, observou-se que os Estados Unidos, com 25 estudos, e o Canadá, com 5 artigos, foram os países onde o tema mais foi investigado, consolidando a América do Norte como principal polo de estudos sobre o assunto. Verificou-se, também, a emergência de pesquisas no continente asiático, com 16 estudos realizados na Índia (4), Taiwan (4), China (3), Israel (3), Irã (1) e Paquistão 
(1). Na Europa foram 10 artigos, três na América Latina (Brasil, México e Peru) e um na Oceania (Austrália), além de dois trabalhos que analisaram o desempenho de polícias, em determinado aspecto, de 77 nações (PARE, 2014) e de 26 países (BARLAGE; VAN DEN BORN; VAN WitTeloostuijn; Graham, 2014). A pesquisa realizada por Wu (2010) na China e nos Estados Unidos foi contabilizada como realizada nos dois países. Trata-se, como visto, de um tema cada vez mais discutido no mundo (SCALCO; AMORIM; GOMES, 2012).

As pesquisas foram divulgadas em periódicos com diversos escopos, em especial nas revistas que publicam estudos relacionados à justiça criminal, políticas e práticas penais, práticas de policiamento, estudos a respeito do crime e análises criminológicas, categorizadas como "Law" no SJR, com 45 artigos. Também foram publicadas cinco pesquisas em periódicos especializados em administração pública, três de administração em geral, assim como em journals que divulgam pesquisas das áreas de informática, logística, economia, ciências sociais, ciências políticas e saúde pública, refletindo a abrangência do tema, que pode ser avaliada por diversas abordagens (BRAGA; MOORE, 2003).

\subsection{Características metodológicas dos artigos revisados}

A maioria das pesquisas utilizou uma abordagem quantitativa, totalizando 54 artigos, refletindo a maior ênfase nas pesquisas direcionadas para as dimensões "uso de recursos de forma eficiente e razoável" e "chamar os infratores para prestar contas", que buscaram avaliar, objetivamente, por meio de estatísticas das atividades policiais, o seu desempenho. Foram encontrados quatro estudos com emprego de abordagem quantitativa e qualitativa. Outros três estudos utilizaram métodos qualitativos, tratando de avaliações de desempenho vinculadas às dimensões "redução do medo e aumento da sensação de segurança" (CAPUTO; MCINTYRE; WANG; HODGKINSON, 2018) e "uso da força e autoridade de forma justa, eficiente e efetiva" (CIHAN, 2014; VILA; JAMES; JAMES, 2018). A Figura 3 evidencia a prevalência da abordagem quantitativa nos estudos revisados. 
Figura 3 | Abordagem utilizada nos artigos revisados

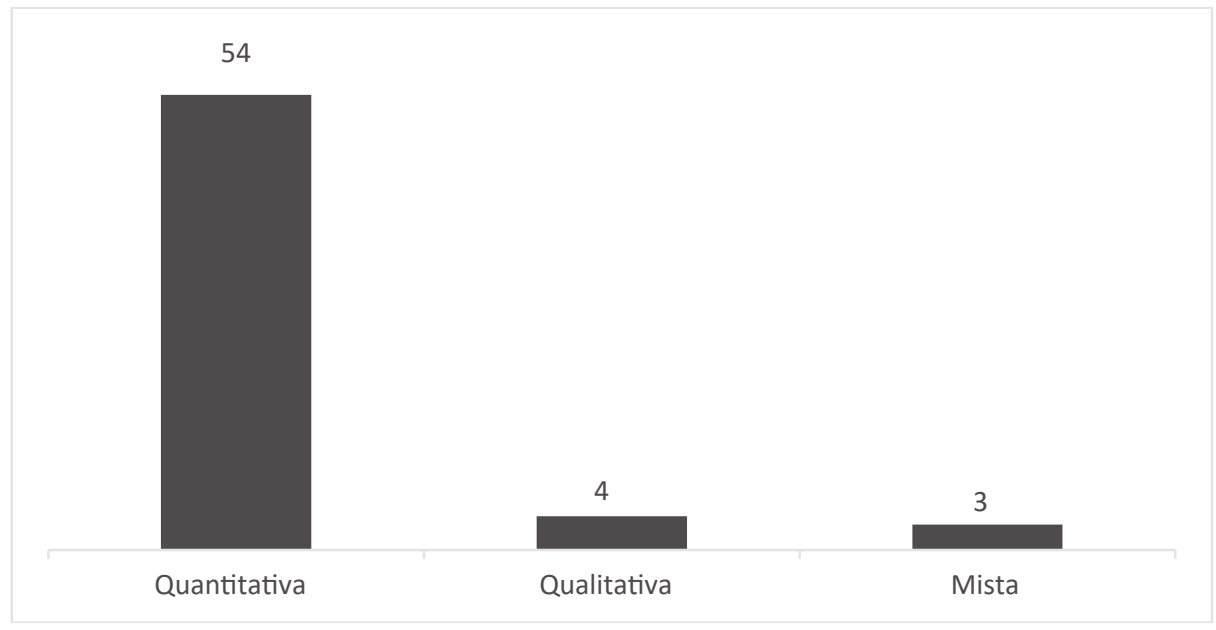

Fonte: elaborado pelos autores.

A pesquisa documental, efetuada em 41 pesquisas, e os questionários, empregados em 23 artigos, foram as principais técnicas de coleta de dados utilizadas, individualmente ou associadas a outros meios de obtenção de informações. As entrevistas foram realizadas em cinco estudos, sempre associadas a outros instrumentos de coleta. Por fim, o grupo focal foi utilizado em três pesquisas, sendo associado à realização de entrevistas no estudo que buscou avaliar o efeito do emprego de tecnologia no policiamento e seus efeitos na redução da criminalidade (KOPER; LUM; HIBDON, 2016).

A técnica de análise de dados mais aplicada foi a regressão, utilizada em 33 artigos, como no trabalho de Roberts (2015), por exemplo, que utilizou regressão logística multinível para estudar taxas de apuração de homicídios. A análise envoltória de dados (DEA) foi empregada em 14 artigos, sendo muito utilizada para avaliar e comparar a eficiência do desempenho entre unidades da polícia (AKDOGAN, 2012; AsIF et al., 2018; SinUANY-Stern; AlPER, 2019). A correlação, em 14 artigos, e a análise fatorial, em nove publicações, completam as técnicas estatísticas mais utilizadas nos estudos quantitativos. Nas pesquisas com abordagem qualitativa não foram informadas as técnicas utilizadas para analisar os dados encontrados. Tal quadro demonstra que as pesquisas que têm por escopo avaliar o desempenho policial aplicam diferentes métodos (VINOD KUMAR, 2014), de acordo com a dimensão e as variáveis utilizadas. 
A análise do desempenho se concentrou no nível organizacional, com 56 artigos, enquanto cinco artigos avaliaram o desempenho em nível individual. Nenhuma pesquisa utilizou o nível de grupo para analisar o desempenho policial. Os estudos que avaliaram o desempenho em nível individual se concentraram, especialmente, em avaliar como as características pessoais dos policiais influenciam os resultados das atividades policiais realizadas (SHANE, 2010, 2013; DESMOND et al., 2020).

Em relação à origem dos dados, 35 pesquisas usaram dados primários, coletados pelos próprios pesquisadores, e 21 artigos utilizaram dados secundários, fornecidos por terceiros (governos, polícias, entre outros). Dos cinco artigos que usaram dados primários e secundários, três empregaram abordagens mistas (qualitativa e quantitativa) para efetuar a pesquisa (LACEY; CugAnesAn; Goode; JACOBS, 2012; Rengifo; Fratello, 2015; KUO; SHIH, 2018).

\subsection{Dimensões de desempenho, variáveis mais utilizadas e principais resultados de pesquisa}

Os artigos revisados não informam expressamente quais são as dimensões avaliadas. Assim, para fins de classificação, foram analisados os objetivos dos estudos e as variáveis empregadas, utilizando as dimensões propostas por Moore e Braga (2003) para catalogálos. Cada uma dessas dimensões invoca uma perspectiva importante que pode e deve ser usada por pesquisadores, gestores e cidadãos para avaliar as organizações policiais. Dos estudos, 40 se concentraram em avaliar apenas uma dimensão, enquanto 21 analisaram duas ou mais dimensões. A Figura 4 sumariza as dimensões examinadas nas pesquisas. 
Figura 4 | Dimensões propostas por Moore e Braga (2003) avaliadas nos estudos revisados

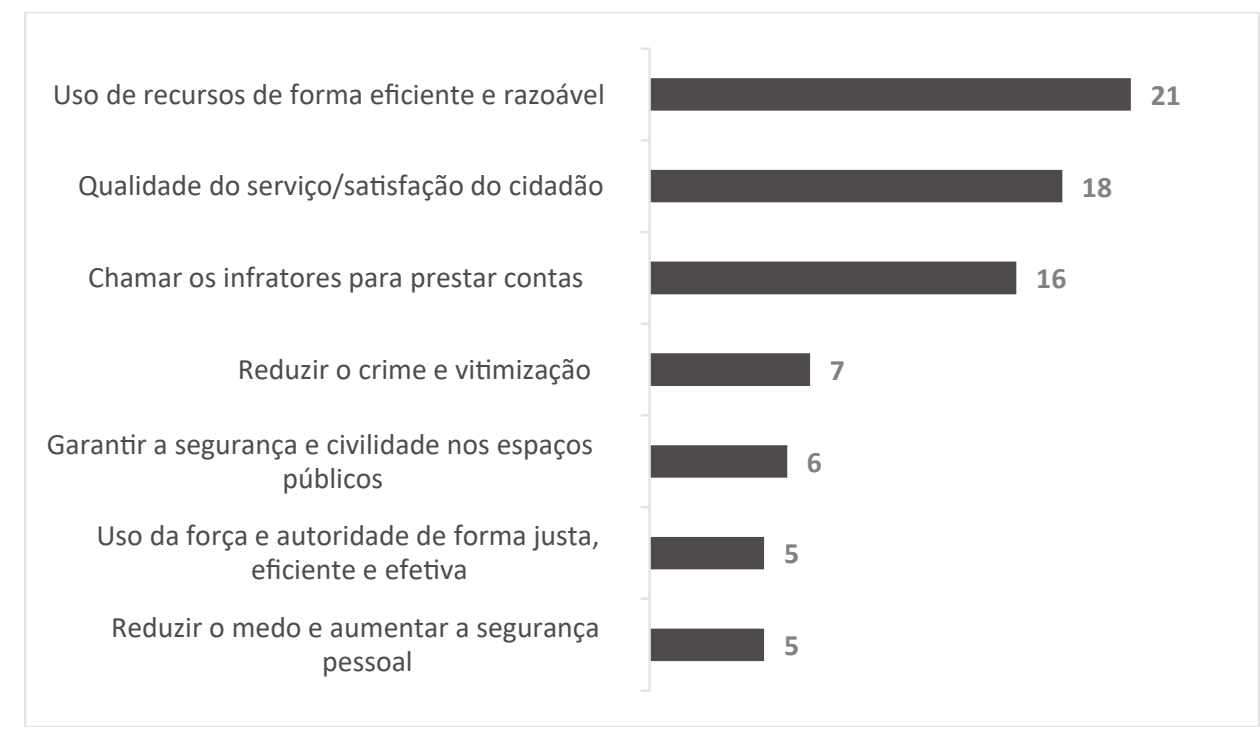

Fonte: elaborado pelos autores.

A dimensão mais estudada nos artigos revisados foi o "uso de recursos de forma eficiente e razoável", com 21 publicações. As atividades realizadas pela polícia são variadas, não sendo fácil desenvolver indicadores para medir sua eficiência (WU; CHEN; YEH, 2010), inclusive porque nem todas as variáveis estão sob o controle da força policial (KUMAR; KUMAR, 2013). Nos estudos revisados, o número de policiais (ARISTOVNIK; SELAK; Mencinger, 2014; FerRANDINo, 2012; VuOREnSYRJÄ, 2018), o número de viaturas (ALDA; DAMMERT, 2019; SINUANY-STERN; ALPER, 2019) e o orçamento da polícia (custos/despesas) (HADAD; KEREN; HANANI, 2015; AgARWAL et al., 2019) foram as variáveis mais utilizadas para medir a sua eficiência.

As pesquisas que abordaram essa dimensão tiveram objetivos e resultados muito diversificados. Kumar e Kumar (2013), por exemplo, constataram que a introdução de dispositivos de comunicação e o aumento das despesas de treinamento ajudaram a melhorar a produtividade dos departamentos de polícia da Índia. Vuorensyrjä (2018) verificou a queda de produtividade da polícia ao longo das reformas gerenciais realizadas na Finlândia. Outras pesquisas tiveram o objetivo de comparar a eficiência de unidades 
policiais (FERRANDINo, 2012; AsIF et al., 2018; AldA; DAMMERT, 2019). No nível individual de desempenho, foi constatado que as variáveis demográficas dos policiais, como gênero e raça (DESMOND et al., 2020), idade, estado civil e o número de filhos (SHANE, 2010, 2013) impactaram a produtividade das organizações avaliadas.

Os resultados das pesquisas em relação ao impacto dos fatores ambientais (exógenos ou externos) na eficiência das polícias também se apresentaram variados. 0 ambiente socioeconômico não influenciou os índices de eficiência nas pesquisas realizadas por Scalco, Amorim e Gomes (2015) e Sinuany-Stern e Alper (2019). No entanto, em diversas pesquisas foram constatados os efeitos de fatores ambientais na eficiência da polícia, como a localização da delegacia (TOMAŽEvič; SELJAK; ARISTOVNIK, 2016), o tamanho da área de circunscrição (AKDOGAN, 2012), a desigualdade de renda (ALDA; DAMMERT, 2019), e o nível de educação da população (RAHIMI et al., 2017; WU; CHEN; YEH, 2010).

A segunda dimensão mais estudada foi "Qualidade do serviço/satisfação do cidadão", com 18 pesquisas. Não é fácil medir a qualidade do serviço público, especialmente o prestado pela polícia, onde os "clientes" são cidadãos vulneráveis, cujo contato com o serviço pode ser limitado (CHEN et al., 2014). Nesse cenário, foi verificado que as principais variáveis utilizadas nas pesquisas que avaliaram a satisfação com o desempenho das polícias foram as características pessoais dos respondentes (WU, 2010; RIDGEWAY; MACDONALD, 2014; Ho; WONHYUK, 2017; LAI; ZHAO, 2018).

Os resultados das pesquisas foram divergentes. As características demográficas foram fracamente relacionadas com a satisfação em alguns estudos (JIANG; SUN; WANG, 2012; BOURANTA; SISKOS; TSOTSOLAS, 2015). No entanto, em outros, características pessoais como raça (RIDGEWAY; MACDONALD, 2014), nível socioeconômico (WU, 2010), nível de escolaridade (LAI; ZHAO, 2018), gênero e idade (HO; WONHYUK, 2017) tiveram impacto na satisfação com a polícia. Hjortskov (2017) constatou que a ordem das perguntas nas pesquisas afeta as avaliações de satisfação.

No desempenho de organizações policiais, compreender as variações da taxa de apuração de crimes é um passo importante para determinar o que pode melhorá-las ao longo do tempo (SCOTT et al., 2019). Neste contexto, "chamar os infratores para prestar contas" foi a terceira dimensão mais utilizada para avaliar o desempenho policial, com 16 
pesquisas. O número de policiais (WEISBURD et al., 2010; DOERNER; DOERNER, 2012; ASIF et al., 2018; AgARWAL et al., 2019; SinUANY-StERn; AlPER, 2019) e o tipo de crimes praticados (Weisburd et al., 2010; Doerner; Doerner, 2012; Hur, 2013; Demers; Rossmo, 2015; KUO; SHIH, 2018; VIDAL; KIRCHMAIER, 2018; SCOTT et al., 2019) foram as variáveis mais empregadas para avaliar essa dimensão de desempenho. Na maioria das pesquisas foi constatado que o maior número de policiais implica mais crimes apurados. No entanto, curiosamente, no estudo feito por Hur (2013) o número de policiais foi negativamente correlacionado com as taxas de apuração de crimes.

Ferrandino (2014) observou que o nível de equidade racial em uma unidade policial não foi um preditor significativo para aumentar a quantidade de crimes apurados. Rumney, McPhee, Fenton e Williams (2019) constataram que unidades especializadas em crimes sexuais (estupros) tiveram melhor desempenho para identificar e prender os criminosos do que as unidades não especializadas. Uma importante constatação de Roberts (2015) é de que as taxas de depuração de crimes devem ser consideradas de acordo com o tipo de ilícito, consoante a dificuldade para realizar a investigação. $O$ aumento do uso da tecnologia também melhora os índices de apuração de crimes (KUMAR; KUMAR, 2013) e facilita a identificação dos criminosos (KovALCHIK et al., 2017). Verificou-se, também, que o tempo entre a prática do crime e o início da atividade investigativa por um detetive pode ser usado como indicador de desempenho na apuração de ilícitos (FALLIK, 2019).

Kuo (2013) observou que, nos locais onde foi instituído o policiamento orientado para a comunidade, a taxa de apuração dos crimes patrimoniais piorou significativamente e dos demais ilícitos não se alterou. Doerner e Doerner (2012) apuraram que não houve diferença nos índices de crimes resolvidos entre organizações policiais que tinham o credenciamento como entidades policiais que realizavam "melhores práticas" e aquelas que não eram credenciadas pela Commission on Accreditation for Law Enforcement Agencies (CALEA).

Um bom policiamento contribui para reduzir a violência (PARE, 2014), mas a polícia não tem controle total sobre muitos fatores que podem impedir ou diminuir os crimes (HADAD; KEREN; HANANI, 2015). Sob essa perspectiva, a quarta dimensão mais estudada foi "Reduzir o crime e a vitimização", com sete artigos que buscaram identificar o desempenho de organizações policiais, avaliando a sua capacidade em reduzir a prática de crimes. 
O número de policiais e a taxa de criminalidade foram as variáveis mais utilizadas, tendo sido constatado que mais policiais implicam menor quantidade de crimes praticados, especialmente os crimes contra a propriedade (FEGLEY; BOSTAPH, 2018; DEMERS, 2019).

Em relação aos resultados, Kuo e Shih (2018) constataram que o emprego do policiamento comunitário não reduziu significativamente a taxa de crimes. Pasha (2018) verificou que o gerenciamento de desempenho é benéfico para diminuir a prática de crimes patrimoniais, mas não reduz crimes "desorganizados", como assassinato e estupro. Shirlow et al. (2013) observaram que a percepção do crime sectário (por motivos religiosos) é muito maior do que o crime registrado.

Koper et al. (2016) estudaram o uso de tecnologia de computação móvel para vigilância e fiscalização e constataram que os aplicativos básicos da computação móvel podem ter pouco ou nenhum impacto direto e mensurável na capacidade dos policiais de reduzir a criminalidade em campo, necessitando de maior treinamento e ênfase nos usos estratégicos da tecnologia para solução de problemas e prevenção de crimes. Fegley e Bostaph (2018) verificaram que comunidades menores têm uma taxa de criminalidade menor que cidades com população maior.

As agências policiais são órgãos essenciais da sociedade, com mandato para manter a ordem e fazer cumprir a lei (PASHA, 2018). "Garantir a segurança e civilidade nos espaços públicos" foi a quinta dimensão mais estudada, com seis pesquisas que avaliaram o desempenho das polícias sob esse aspecto. Os quatro estudos que avaliaram o desempenho da polícia em relação à segurança do trânsito utilizaram, principalmente, o número de policiais e de viaturas e as características ambientais (densidade populacional, área da circunscrição, quantidade de veículos, características das vias públicas) como variáveis independentes para avaliar os incidentes de trânsito (AKDOGAN, 2012; ARISTOVNIK; SELAK; Mencinger, 2013; RAHIMI et al., 2017; SinUANY-SteRn; AlPER, 2019).

No estudo que empregou como variável o efeito de programa de policiamento comunitário na manutenção da ordem no bairro, foi verificado que, nos bairros que receberam o programa, os cidadãos estavam mais contentes com a atuação da polícia nesse aspecto (LOMBARDo, OLSON; STATON, 2010). Zhao et al. (2014) constataram que as percepções a respeito da desordem na vizinhança não tinham impacto na avaliação dos residentes a respeito da polícia local. 
A polícia é definida como um corpo constituído de cidadãos habilitados pelo Estado a fazer cumprir a lei, proteger a propriedade e limitar a desordem civil por meio do uso legitimado da força (AGARWAL et al., 2019), o que pode requerer o uso de violência física ou verbal (GARcíA-SÁnchez; Rodríguez-Domínguez; PARRA-Domínguez, 2013), devendo ser feito bom uso dos poderes coercitivos (MADAN; NALLA, 2015). Avaliações positivas da polícia local podem ser corroídas pela exposição à discriminação e práticas policiais mais intensas (RENGIFO; Fratello, 2015). A dimensão "uso da força e autoridade de forma justa, eficiente e efetiva", que avalia o desempenho policial com essas premissas, foi uma das menos pesquisadas, com cinco estudos.

Nas pesquisas que se concentraram nessa dimensão, as variáveis mais utilizadas foram as características pessoais dos respondentes e as características da vizinhança. Contrariando as suposições dos autores, que esperavam uma menor atenção da polícia, verificou-se que a atuação policial não discriminou os bairros e as pessoas mais pobres, agindo de forma justa ao prestar o serviço, concedendo até uma atenção superior a outros bairros menos desfavorecidos (CIHAN, 2014; 2015). No entanto, estudantes chineses não acreditavam que a polícia local tratasse igualmente os cidadãos ricos e pobres (WU; SUN, 2010). Em uma das pesquisas foi desenvolvida uma escala para avaliar a força utilizada em encontros com a polícia (VILA; JAMES; JAMES, 2018).

Os serviços policiais devem se preocupar não apenas com o crime, mas também com elementos como o medo do crime (BOURANTA; SISKOS; TSOTSOLAS, 2015), que compõem a dimensão "Reduzir o medo e aumentar a segurança pessoal", estudada em cinco artigos. Para pesquisar essa dimensão, as variáveis mais utilizadas foram as características pessoais dos cidadãos e a vitimização de crimes, sendo constatado que os atributos pessoais dos respondentes dos questionários, especialmente idade e gênero (BARRETT; PEIRONE; CHEUNG, 2019), e suas experiências como vítimas de crimes, impactaram o medo e o baixo nível de sensação de segurança (BOIVIN; CORDEAU, 2014).

A comparação entre os locais com e sem policiamento comunitário mostra tendências semelhantes em relação à sensação de segurança das pessoas, o que pode ser atribuído ao fato de que a acessibilidade à polícia e a sensação de segurança são afetadas pelas características dos membros da comunidade, não se constatando, portanto, variação nesse aspecto nos diferentes mecanismos de prestação de serviços policiais (VINOD KUMAR, 
2014). Em relação a algumas dessas características pessoais, no caso o gênero, verificou-se que os homens que tinham mais confiança na polícia exibiram maior percepção de medo do crime do que as mulheres nas áreas de perigo em espaços públicos, enquanto que as mulheres que informaram baixos níveis de confiança na polícia obtiveram pontuação mais alta em relação ao medo do crime nesses locais (MARTínEZ-FERRER et al., 2018).

Por meio da síntese dos estudos que foi apresentada, percebe-se que o desempenho de organizações policiais é um assunto importante e vasto, cuja produção acadêmica foi fértil nos últimos 10 anos. É um tema que requer aprofundamentos e delineamentos, dada a complexidade e a diversidade dos serviços prestados pelas organizações policiais, que tornam difícil o estabelecimento de metodologia única para que seja realizada a avaliação do seu desempenho. Por essa razão, o desempenho de organizações policiais deve ser mensurado de acordo com os objetivos e atribuições de cada polícia que será avaliada, conforme as atividades que são realizadas e em consonância com a dimensão que se deseja medir. Somente após essa definição é possível escolher as variáveis que serão utilizadas para analisá-lo.

\section{Considerações finais}

O aprofundamento dos estudos a respeito do desempenho das polícias é fundamental para o desenvolvimento e a consolidação de conhecimentos que contemplem as peculiaridades desse tipo de organização, o que justifica a preocupação com uma teorização específica, que contemple outros aspectos além dos já conhecidos nos estudos sobre o desempenho organizacional de outras entidades públicas e privadas.

Por meio da revisão sistemática da literatura realizada, foi possível constatar que o fenômeno desempenho policial tem sido muito pesquisado no mundo, especialmente na América do Norte, Europa e Ásia. O tema foi objeto de variados estudos ao longo da última década, com o emprego de diferentes metodologias e abordagens. Os artigos analisados reproduziram a complexidade, heterogeneidade e diversidade do desempenho policial (CARTER; KLEIN; DAY, 1993; VolLARD, 2003), refletindo os diferentes contextos e atividades desempenhadas pelas diversas polícias que foram objeto dos estudos. 
Diante de tal complexidade, verifica-se que não existe uma única teoria sobre o desempenho policial, mas, sim, muitas maneiras para estudá-lo. Assim, o caminho para realizar pesquisas sobre o fenômeno é identificar conjuntos de variáveis específicas, aplicadas às atividades realizadas pelas polícias, de acordo com as suas atribuições e peculiaridades (DOOREN, 2005), consoante a dimensão do desempenho que se pretenda estudar (MASLOV, 2014).

Com base nessas considerações, se propõe uma agenda de pesquisa voltada para definir o desempenho das polícias brasileiras e avaliá-lo, de acordo com as suas características e atribuições. Faz-se necessário estudar o desempenho das polícias nacionais, nas suas diversas dimensões, buscando compreender as atividades realizadas e identificar como elas podem ser mensuradas, de modo a aumentar o controle social sobre essas organizações e melhorar o seu desempenho.

Nesse sentido, este trabalho traz uma contribuição significativa para um campo de conhecimento que carece de estudos empíricos no Brasil. Foram apresentados estudos, as suas características metodológicas, principais variáveis utilizadas e resultados, que podem auxiliar em pesquisas voltadas para a elaboração e testagem de indicadores do desempenho das polícias brasileiras, o que permitiria o aprimoramento das suas ações, resultando na racionalização de recursos públicos e na melhoria dos serviços prestados por tais instituições.

\section{Referências}

Agarwal, P.; Hunt, K.; ZhuAng, J.; SARKAR, B.; SARkaR, A.; Sharma, R. An exploratory analysis for performance assessment of state police forces in Índia: an eclectic approach. Operational Research, 2019.

AKDOGAN, H. The efficiency of police stations in the city of Ankara: an application of data envelopment analysis. Policing: An International Journal of Police Strategies \& Management, v. 35, n. 1, p. 25-38, 2012.

ALDA, E.; DAMMERT, L. Weathering the Storm! The effects of the external environment on police efficiency in Peru. Policing: An International Journal of Police Strategies \& Management, v. 42, n. 6, p. 1124-1140, 2019. 
ARISTOVNIK, A.; SELJAK, J.; MENCINGER, J. Performance measurement of police forces at the local level: a non-parametric mathematical programming approach. Expert Systems with Applications, v. 41, n. 4 , p. $1647-1653,2014$.

ARISTOVNIK, A.; SELJAK, J.; MENCINGER, J. Relative efficiency of police directorates in Slovenia: a non-parametric analysis. Expert Systems with Applications, v. 40, n. 2, p. 820-827, 2013.

AsIF, M.; ShahZAD, M.; AWAN, M. U.; AKDogan, H. Developing a structured framework for measuring police efficiency. International Journal of Quality and Reliability Management, v. 35, n. 10, p. 2119-2135, 2018.

BAKKALBASI, N.; BAUER, K.; GLOVER, J.; WANG, L. Three options for citation tracking: Google Scholar, Scopus, and Web of Science. Biomed. Digit. Libr. v. 3, n. 7, 2016.

Barlage, M.; Van Den Born, A.; Van Witteloostuijn, A.; Graham, L. Estimating public performance bias through an MTMM model the case of police performance in 26 European countries. Policy Studies, v. 35, n. 4, p. 377-396, 2014.

Barrett, B. J.; Peirone, A.; Cheung, C. H. Spousal violence and evaluations of police performance in Canada: does police contact matter? Journal of Family Violence, v. 34 n. 3, p. 199-211, 2019.

BoIvin, R.; CORDEAU, G. Do Web surveys facilitate reporting less favourable opinions about law enforcement? Security Journal, v. 30, n. 2, p. 335-348, 2014.

BOURANTA, N.; SISKOS, Y.; TSOTSOLAS, N. Measuring police officer and citizen satisfaction: comparative analysis. Policing: An International Journal of Police Strategies \& Management, v. 38, n. 4, p. 705-721, 2015.

CANO, I.; DUARTE, T. L. As corregedorias dos órgãos de segurança pública no Brasil. Revista Brasileira de Segurança Pública, v. 8, n. 2, p. 84-108, 2014.

CAPUto, T.; MCINTYRe, M. L.; WANG, L. M. Y.; Hodgkinson, T. Assessing what police officers do "on the job": toward a "public values" approach. Policing: An International Journal of Police Strategies \& Management, v. 41, n. 1, p. 70-83, 2018.

CARMONA, S.; GRÖNLUND, A. Measures vs actions: the balanced scorecard in Swedish law enforcement. International Journal of Operations \& Production Management, v. 23, n. 12, p. 1475-1496, 2003.

Coordenação de Aperfeiçoamento de Pessoal de Nível Superior (CAPes). Critérios de classificação Qualis-Ensino, n.d. Recuperado em 30 de abril de 2020, de https://www.capes.gov. $\mathrm{br} /$ images/stories/download/avaliacaotrienal/Docs_de area/qualis/ensino.pdf.

CARTER, N.; KLEIN, R.; DAY, P. How organizations measure success: the use of performance indicators in government. London: Routledge, 1993.

CHEN, C. M.; LEE, H. T.; CHEN, S. H.; TSAI, T. H. The police service quality in rural Taiwan: a comparative analysis of perceptions and satisfaction between police staff and citizens, Policing: An International Journal of Police Strategies \& Management, v. 37, n. 3, p. 521-542, 2014. 
CIHAN, A. Social disorganization and police performance to burglary calls: a tale of two cities. Policing: An International Journal of Police Strategies \& Management, v. 37, n. 2, p. 340-354, 2014.

CiHAN, A. Examining the neighborhood effects on police performance to assault calls. Police Practice and Research, v. 16, n. 5, p. 391-401, 2015.

COLLIER, P. Operations and accountability: the role of performance indicators, financial devolution and strategy in the management of a police force. International Journal of Police Science and Management, v. 1, n. 1, p. 81-93, 1998.

COLLIER, P. The power of accounting: a field study of local financial management in a police force. Management Accounting Research, v. 12, p. 465-86, 2001.

DAVIS, R. C. Selected international best practices in police performance measurement. Santa Monica, CA: RAND Corporation, 2012.

DemERS, S. More canadian police means less crime. Canadian Journal of Criminology and Criminal Justice, v. 61, n. 4, p. 69-100, 2019.

DEMERS, S.; Rossmo, D. Simpson's paradox in canadian police clearance rates. Canadian Journal of Criminology and Criminal Justice, v. 57, n. 3, p. 424-434, 2015.

Desmond, J. S.; Reyns, B. W.; Frank, J.; Klahm IV, C. F.; Henson, B. Police productivity and performance over the career course: a latent class growth analysis of the first 10 years of law enforcement. Police Quarterly, 2020.

Doerner, W. M.; Doerner, G. M. Police accreditation and clearance rates. Policing: An International Journal of Police Strategies \& Management, v. 35, n. 1, p. 6-24, 2012.

DOOREN, W. What makes organizations measure? Hypotheses on the causes and conditions for performance measurement. Financial Accountability \& Management, v. 21, n. 3, p. 363-383, 2005.

DRAKE, L. M.; SIMPER, R. Police efficiency in offences cleared: an analysis of English "Basic command units". International Review of Law and Economics, v. 25, p. 186-208, 2005.

DURANTE, M.; Borges, D. Avaliação e desempenho em segurança pública. In: Indicadores de Desempenho em Segurança Pública. Brasília, DF: Ministério da Justiça, p. 63-86, 2011.

Encontro nacional da Associação Nacional de Pós-Graduação e Pesquisa em Administração (ENANPAD),2020. Recuperado em 06 de setembro de 2020, de http://www.anpad.org.br/files up/2020/1/106/20200129143038-Chamada_Trabalhos_EnANPAD_PO_OF_.pdf.

FALLIK, S. W. How long does it take? An exploration of the time between case assignment and a detective's first investigative activity. Police Practice and Research, v. 20, n. 2, p. 185-200, 2019.

FEGLEY, T.; BOSTAPH, L. G. Is bigger better? An analysis of economies of scale and market power in police departments. Policing: An International Journal of Police Strategies \& Management, v. 41, n. 5, p. 578-592, 2018. 
FERRANDINO, J. The comparative technical efficiency of Florida campus police departments. Criminal Justice Review, v. 37, n. 3, p. 301-318, 2012.

FERRANDINO, J. The relationship between police minority equity levels and performance outcomes. Journal of Ethnicity in Criminal Justice, v. 12, n. 2, p. 69-92, 2014.

Ferreira, B. A. A.; Rigueira, A. L. Os indicadores-chave de desempenho como aliados da análise criminal. Revista Brasileira de Segurança Pública, v. 7, n. 2, p. 68-88, 2013.

FINK, A. Conducting research literature reviews: from paper to the internet, Sage Publications, London, 2014.

Gallagher, C.; Maguire, E. R.; Mastrofski, S. D.; Reisig, M. D. The Public Image of the Police. Final Report to the International Association of Chiefs of Police by the Administration of Justice Program, George Mason University, 2001.

García-SÁnchez, I. M.; Rodríguez-Domínguez, L.; PARRA-Domínguez, J. Evaluation of the efficacy and effectiveness of the Spanish security forces. European Journal of Law and Economics, v. 36, n. 1, p. 57-75, 2013.

González-Pereira, B.; Guerrero-Bote, V. P.; MoyA-Anegón, F. A new approach to the metric of journals scientific prestige: the SJR indicator. Journal of Informetrics, v. 4, n. 3, p. 379-391, 2010.

HADAD, Y.; KeREN, B.; HANANI, M. Z. Combining data envelopment analysis and Malmquist Index for evaluating police station efficiency and effectiveness. Police Practice and Research: An International Journal, v. 16, n. 1, p. 5-21, 2015.

HJORTSKOV, M. Priming and context effects in citizen satisfaction surveys. Public Administration, v. 95, n. 4, p. 912-926, 2017.

Ho, A. T.; WonhyUK, C. Government communication effectiveness and satisfaction with police performance: a large-scale survey study. Public Administration Review, v. 77, n. 2, p. 228-239, 2017.

HOLMES, J.; PIÑERES, S. G.; KIEL, D. Reforming government agencies internationally: is there a role for the balanced scorecard? International Journal of Public Administration, v. 29, n. 12, p. 112545, 2006.

Hoque, Z.; Arend, S.; Alexander, R. Policing the police service: a case study of the rise of "new public management" within an Australian police service. Accounting, Auditing \& Accountability Journal, v. 17, n. 1, p. 59-84, 2004.

HUR, Y. Racial diversity, is it a blessing to an organization? Examining its organizational consequences in municipal police departments. International Review of Administrative Sciences, v. 79, n. 1, p. 149-164, 2013.

JIANG, S.; SUN, I. Y.; WANG, J. Citizens' satisfaction with police in Guangzhou, China. Policing: An International Journal of Police Strategies \& Management, v. 35, n. 4, p. 801-821, 2012.

Kiedrowski, J.; Petrunik, M.; Macdonald, T.; Melchers, R. Canadian Police Board Views on the Use of Police Performance Metrics. Ottawa: Public safety Canada, 2013. 
KOPER, C. S.; LUM, C.; HIBDON, J. The uses and impacts of mobile computing technology in hot spots policing. Evaluation Review, v. 39, n. 6, p. 587-624, 2016.

Kovalchik, S. A.; Herberman, E.; MugG, K.; Jackson, B. A. Developing outcome measures for criminal justice information sharing: a study of a multi-jurisdictional officer notification system for policing sex offenders in Southern California. American Journal of Criminal Justice, v. 42, n. 2, p. 275-291, 2017.

KUMAR, S.; KUMAR, S. Does modernization improve performance: evidence from Indian police. European Journal of Law and Economics, v. 39, n. 1, p. 57-77, 2013.

KUO, S.; SHIH, Y. An evaluation of a Community-Oriented Policing Program in Taiwan. International Journal of Offender Therapy and Comparative Criminology, v. 62, n. 7, p. 2016-2044, 2018.

LACERDA, R. T.; ENSSLIN, L.; ENSSLIN, S. R. Uma análise bibliométrica da literatura sobre estratégia e avaliação de desempenho. Gestão e Produção, v. 19, n. 1, p. 59-78, 2012.

LACEY, D.; CUGANESAN, S.; GOODE, S.; JACOBS, K. Celebrating adversity: inter-organizational dependence and public sector performance reporting in the Australian federal police. Public Administration, v. 90, n. 2, p. 393-411, 2012.

LAI, Y.; ZHAO, R. The impacts of neighborhood context on residents' satisfaction with police services in metropolitan Taipei: a multilevel approach. Policing: An International Journal of Police Strategies \& Management, v. 41, n. 2, p. 276-291, 2018.

LEE, J.; LEE, J.; HOOVER, L. T. What conditions affect police response time? Examining situational and neighborhood factors. Police Quarterly, v. 20, n. 1, p. 61-80, 2017.

LELANDAIS, I. Mesure de la performance policière: expériences internationales, Montréal, Centre international pour la prévention de la criminalité, 2007.

LOMBARDO, R. M.; OLSON, D.; STATON, M. The Chicago alternative policing strategy: a reassessment of the CAPS program. Policing: An International Journal of Police Strategies \& Management, v. 33, n. 4, p. 586-606, 2010.

MADAN, M.; NALLA, M. K. Exploring citizen satisfaction with police in India: the role of procedural justice, police performance, professionalism, and integrity. Policing: An International Journal of Police Strategies \& Management, v. 38, n. 1, p. 86-101, 2015.

MAgUiRe, E. R. Police departments as learning laboratories. Ideas in American Policing, n. 6. Washington, DC: Police Foundation, 2004.

MAILLARD, J.; SAVAGE, S. P. Comparing performance: the development of police performance management in France and Britain. Policing and Society: An International Journal of Research and Policy, v. 22, n. 4, p. 363-383, 2012.

MAILlARD, J.; SAVAGE, S. P. Policing as a performing art? The contradictory nature of contemporary police performance management. Criminology \& Criminal Justice, v. 18, n. 3, p. 314-331, 2018. 
Martínez-Ferrer, B.; Vera, J. A.; Musitu, G.; Montero-Montero, D. Trust in police and fear of crime among young people from a gender perspective: the case of Mexico. Violence and Gender, v. 5, n. 4, p. 226-232, 2018.

MASLOV, A. Measuring the performance of the police: the perspective of the public. Public Safety Canada, 2014.

MIRzer, M. L. Policing supervision in the 21st century. FBI Law Enforcement Bulletin, v. 65, p. 6-10, 1996.

Moore, M. H.; BRAGA, A. The "Bottom Line" of policing: what citizens should value (and measure!). Police Performance, Police Executive Research Forum, 2003.

NEYRoud, P. Past, present and future performance: lessons and prospects for the measurement of police performance, Policing: a Journal of Policy and Practice, v. 2, n. 3, p. 340-348, 2008.

PARE, P. Indicators of police performance and their relationships with homicide rates across 77 nations. International Criminal Justice Review, v. 24, n. 3, p. 254-270, 2014.

PASHA, O. Can performance management best practices help reduce crime? Public Administration Review, v. 78, n. 2, p. 217-227, 2018.

PINC, T. Desempenho policial: treinamento importa? Revista Brasileira de Segurança Pública, v. 3, n. 1, p. 36-55, 2009.

Rahimi, H.; Soori, H.; Nazari, S. S. H.; Motevalian, S. A.; Azar, A.; Momeni, E.; Javartani, M. The relative efficiency of Iranian's rural traffic police: a three-stage DEA model. BMC Public Health, v. 17, n. 1, p. 1-9, 2017.

Rengifo, A.; Fratello, J. Perceptions of the police by immigrant youth: looking at stop-and-frisk and beyond using a New York City sample. Youth Violence and Juvenile Justice, v. 13, n. 4, p. 409427, 2015.

RIDGEWAY, G.; MACDONALD, J. M. A method for internal benchmarking of criminal justice system performance. Crime and Delinquency, v. 60, n. 1, p. 145-162, 2014.

ROBERTS, A. Adjusting rates of homicide clearance by arrest for investigation difficulty: modeling incident- and jurisdiction-level obstacles. Homicide Studies, v. 19, n. 3, p. 273-300, 2015.

RoCHE, S. Performance management in France: a police or an electoral issue? Policing: A Journal of Policy and Practice, v. 2, n. 3, p. 331-339, 2008.

ROGERSON, P. Performance measurement and policing: police service or law enforcement agency? Public \& Money Management, v. 15, n. 4, p. 25-30, 1995.

Rosenbaum, D. P.; LaWrence, D. S.; Hartnett, S. M.; MCDeVitT, J.; Posick, C. Measuring procedural justice and legitimacy at the local level: the police-community interaction survey. Journal of Experimental Criminology, v. 11, n. 3, p. 335-366, 2015.

Rumney, P. N. S.; MCPhee, D.; Fenton, R. A.; Williams, A. A police specialist rape investigation unit: a comparative analysis of performance and victim care. Policing and Society, 2019. 
SCALCO, P. R.; AMORIM, A. L.; Gomes, A. P. Eficiência técnica da polícia militar em Minas Gerais. Nova Economia, v. 22, n. 1, p. 165-190, 2012.

SCOTT, T. L.; WeLLFORD, C.; LUM, C.; VoVAK, H. Variability of crime clearance among police agencies. Police Quarterly, v. 22, n. 1, p. 82-111, 2019.

SHANE, J. M. Organizational stressors and police performance. Journal of Criminal Justice, v. 38, n. 4, p. 807-818, 2010.

SHANE J. M. Daily work experiences and police performance. Police Practice and Research, v. 13, n. 3, p. 1-19, 2013.

Shirlow, P.; TAYlor, L. K.; Merrilees, C. E.; Goeke-Morey, M. C.; Cummings, E. M. Hate crime: record or perception? Space and polity, v. 17, n. 2, p. 237-252, 2013.

SinUANY-STERN, Z.; ALPER, D. Factors affecting police station efficiency: DEA in police logistics. International Journal of Logistics Systems and Management, v. 34, n. 1, p. 75-101, 2019.

SouzA, R. V.; REIS, G. P. O controle da polícia no Brasil: uma introdução ao controle científico da polícia. São Paulo em Perspectiva, v. 21, n. 1, p. 76-91, 2007.

TIWANA, N.; BASS, G.; FARREL, G. Police performance measurement: an annotated bibliography. Crime Science, v. 4, n. 1, p. 1-28, 2015.

TOMAŽEVIČ, N.; SELJAK, J.; ARISTOVNIK, A. TQM in public administration organizations: an application of data envelopment analysis in the police service. Total Quality Management and Business Excellence, v. 27, n. 11-12, p. 1396-1412, 2016.

TRANFIELD, D.; DENYER, D.; SMART, P. Towards a methodology for developing evidence-informed management knowledge by means of systematic review. British Journal of Management, v. 14, p. 207-222, 2003.

TYLER, T. R. Public trust and confidence in legal authorities: what do majority and minority group members want from the law and legal authorities? Behavioral Science and the Law, v. 19, n. 2, p. 215-235, 2001.

VIDAL, J. B. I.; KIRCHMAIER, T. The effect of police response time on crime clearance rates. Review of Economic Studies, v. 85, n. 2, p. 855-891, 2018.

VILA, B.; JAMES, S.; JAMES, L. How police officers perform in encounters with the public: measuring what matters at the individual level. Policing: An International Journal of Police Strategies \& Management, v. 41, n. 2, p. 215-232, 2018.

VINOD KUMAR, T. K. Differing services, rising expectations, and greater demands: patterns in variations of police-public dynamics across areas with conventional and community policing in India. Policing: An International Journal of Police Strategies \& Management, v. 37, n. 1, p. 170189, 2014.

VolLARD, B. Performance contracts for police forces. CPB Netherlands Bureau for Economic Policy Analysis, 2003. 
VIEIRA, R.; Protásıo, G. Gestão para resultados na segurança pública em Minas Gerais: uma análise sobre o uso de indicadores na gestão da Polícia Militar e no Sistema de Defesa Social. Revista Brasileira de Segurança Pública, v. 5, n. 8, p. 206-220, 2011.

VUORENSYRJÄ, M. Police management reform, labor productivity, and citizens' evaluation of police services. Policing: An International Journal of Police Strategies \& Management, v. 41, n. 6, p. 749-765, 2018.

WeISBURD, D.; HASISI, B.; JONATHAN, T.; AvIV, G. Terrorist threats and police performance. British Journal of Criminology, v. 50, n. 4, p. 725-747, 2010.

WU, T. H.; CHEN, M. S.; YEH, J. Y. Measuring the performance of police forces in Taiwan using data envelopment analysis. Evaluation and Program Planning, v. 33, n. 3, p. 246-254, 2010.

Wu, Y. College students' evaluation of police performance: a comparison of Chinese and Americans. Journal of Criminal Justice, v. 38, n. 4, p. 773-780, 2010.

Wu, Y.; Sun, I. Y. Perceptions of police: An empirical study of Chinese college students. Policing: An International Journal of Police Strategies \& Management, v. 33, n. 1, p. 93-113, 2010.

ZHAO, J. S.; TSAI, C.; REN, L.; LAI, Y. Public satisfaction with police control of disorder crime: does the public hold police accountable? Justice Quarterly, v. 31, n. 2, p. 394-420, 2014.

\section{Andersson Pereira dos Santos}

(iD) https://orcid.org/0000-0002-1266-1418

Doutorando em Administração e Mestre em Administração Pública pela Universidade de Brasília. Especialista em Gestão de Pessoas no serviço público pela Escola Nacional de Administração Pública. Delegado de Polícia Federal.

E-mail: andersson.aps@pf.gov.br

\section{Adalmir de Oliveira Gomes}

(iD https://orcid.org/0000-0001-9000-3296

Doutor em Administração pela Universidade de Brasília e pós-doutor pela Universidade de Indiana (EUA). Professor Adjunto do Departamento de Administração e do Programa de Pós-Graduação em Administração (PPGA) da UnB.

E-mail: adalmir@unb.br 